\title{
Investigation of the Performance of Multiple Modeling and Control Approach Using a Laboratory Helicopter
}

\author{
V.Nazarzehi, A.Fatehi and M.Zamanian
}

\begin{abstract}
The multiple modeling and control approach is a proper method for modeling and control of nonlinear systems which their dynamics changes rapidly at different operating conditions. The dynamic of the twin rotor helicopter in vertical direction is nonlinear and changes instantaneously with respect to change of the elevation angle, and it can be used for implementation of multiple modeling and control approach. In this paper the performance of multiple modeling and control approach by simulation and implementation has been investigated.
\end{abstract}

Index Terms-Twin rotor helicopter, Multiple modeling, Multiple control

\section{INTRODUCTION}

Most processes have nonlinear and complex structure and their dynamical behavior changes in two ways. They are nonlinear, i.e. their dynamic behavior changes according to variation of operating conditions or they are time variant, i.e. their dynamical behavior changes over time. In both cases a single linear model for a specific operating point could not be a good description of process in full operating range or at each instant of time. The conventional solution is conservative controller tuning for worst case conditions. However, this approach can result in poor control system performance for more typical conditions. Alternatively, adaptive control strategies are available where the controller parameters and/or control structure are modified online as conditions change (Astrom \&Wittenmark, 1995). A special class of adaptive control strategies referred to as multimodel control (Angeli \& Mosca, 2002; Freidovich\& Khalil, 2003; Hespanha, 2001; Johansen \& Murray-Smith, 1997; Morse, 1996; Narendra \& Balakrishnan,1997). Generally The Multiple Modeling and Control approach is a proper method for modeling and control of nonlinear systems which their dynamics changes instantaneously at different operational conditions. The capabilities of multi-model controller have been successfully demonstrated in many applications. Among them are drug infusion control where variability and unpredictability are key issues (He,Kaufman,\& Roy, 1986; Schott \& Bequette, 1997),

V.Nazarzehi is with the Chabahar Maritime University, Chabahar, Iran (corresponding author to provide phone: 0098-545-2220020; fax: 00985452220023; e-mail: nazarzehi@cmu.ac.ir).

A.Fatehi is with the K.N.Toosi University, Tehran,Iran(e-mail: fatehi@kntu.ac.ir).

M.Zamanian is with the K.N.Toosi University, Tehran,Iran(e-mail: mzamanian@kntu.ac.ir). control of $\mathrm{pH}$ neutralization plant (Dougherty \&Cooper, 2003b; Galan, Romagnoli, \& Palazoglu, 2004, Peyman et al 2008), distillation columns (Dougherty \& Cooper, 2003a; Rodriguez, Romagnoli,\& Goodwin, 2003), power systems (Chadouri, Majumder, \& Pal, 2004), and chemical reactors(Tian \& Hoo, 2005)[1],[2],[3].

In order to apply the multiple model controller method, linear model of the plant's dynamical behavior in different operational conditional is required. This can be done in two ways; identification or first principle modeling. A controller is designed according to each of the obtained models. Then, a suitable controller could be selected in every instant of time to control the plant. Although Adaptive controller may also be used in this condition but because of delay in adaptation process to the new situation it has not a suitable performance for modeling and control of processes with rapid changes [4].In this paper multiple modeling and control approach has been used to control a laboratory helicopter. CE150 laboratory helicopter is designed for the theoretical study and practical investigation of basic and advanced control engineering principles, dynamics modeling, identification, and various controllers design by classical and modern methods. Figure 1 displays this twin rotor helicopter. It consists of a body, carrying two propellers driven by DC motors, and a massive support. The body has two degrees of freedom. Both bodies position angles (elevation and azimuth) are influenced by rotation of propellers. The axes of the body rotation are perpendicular. DC motors are driven by power amplifiers using pulse width modulation.

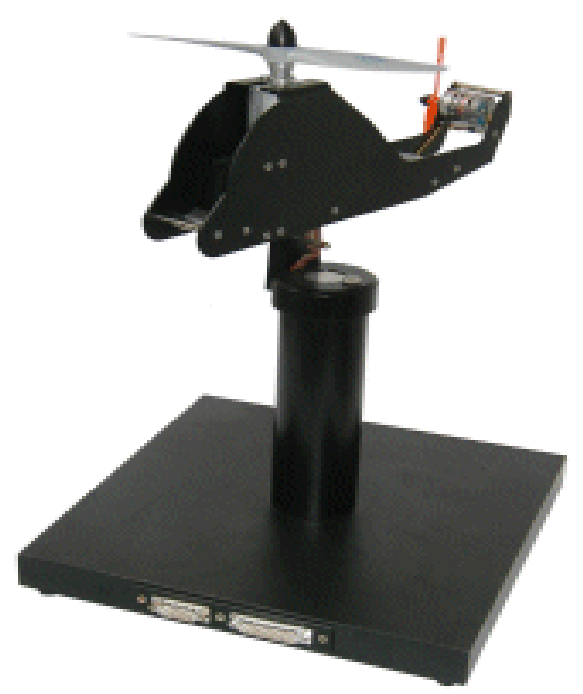

Figure 1. CE 150 laboratory helicopter 
Centre of gravity is changed by moving small weight along the main horizontal axis of helicopter by a servomotor. The helicopter is a multi input multi output (MIMO) dynamical system but its motion can be limited in one direction to make a single input single output (SISO) plant. Generally, processes dynamical behavior may change slowly or instantaneously [5],[6]. Dynamical behavior of this laboratory helicopter can be changed instantaneously in two ways:

- Moving small weight

- Changing Elevation angle

Since the effect of elevation angle variation is more than small weight movement, thus it can be used for changing laboratory helicopter dynamic. The vertical channel of the helicopter is nonlinear and its dynamic change with variation of elevation angle. In this paper the performance of the multiple modeling and control approach by using variation of elevation angle (operating point) has been investigated.

This paper is organized as follows. In section 2 multiple models of the helicopter are obtained in different operating points. In section 3, the multiple controllers are designed using multiple models. In section 4 multiple modeling and control approach has been described. Finally in sections 5 and 6 the multiple modeling and control approach is first simulated and then implemented on the helicopter and compared with different methods.

\section{MULTIPLE MODEL OF THE LABORATORY HELICOPTER}

In many controller design methods a mathematical model of plant is required in order to explain a dynamical behavior of plant and it can be used to design a controller. There are two well known modeling approaches: Theoretical and Experimental.. The first method is known as the first principle modeling. According to this technique, the model can be obtained by mathematical equations which are based on physical laws. The second one is system identification, according to system identification technique by assuming a plant as a black box and applying some test on the plant and considering a suitable model structure, the parameters of the model can be estimated. In this research, suitable multiple models are obtained for elevation direction of the helicopter based on first principle modeling and their performances are investigated.

\section{A. Laboratory helicopter dynamic modeling}

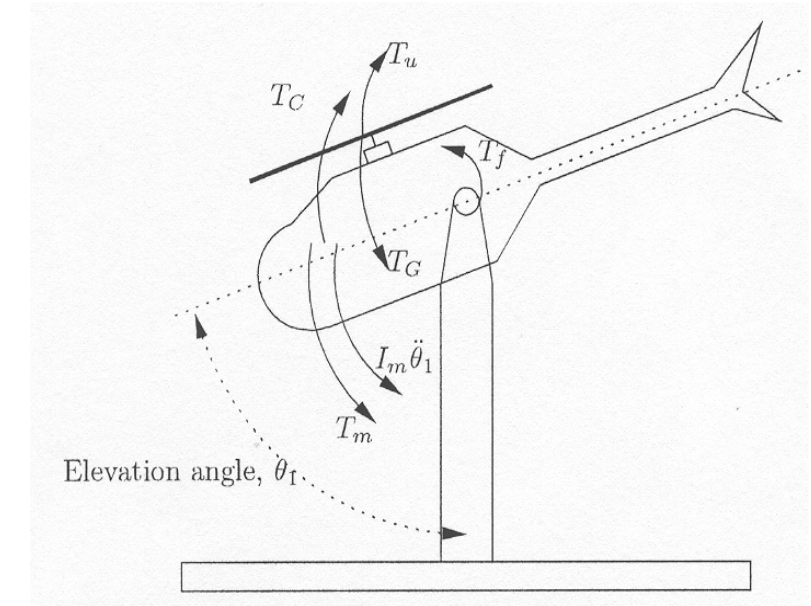

Figure 2: torques equilibrations in vertical plane

The plant's dynamic equation is obtained with writing torques equilibrations from figure 2 and combining them with DC motor dynamic equations. Torques equilibrations in vertical plane can be expressed as follow [5]:

$$
I_{m} \theta_{1} \cdot=T_{u}-T_{f}-T_{m}
$$

where $T_{u}=k \omega^{2}$,

$$
T_{m}=T_{g} \sin \left(\theta_{1}+\alpha\right) \text { and } T_{f}=\left(\beta_{11}|\omega|+\beta_{21}\right) \theta_{1}^{\bullet}
$$

The Dynamical behavior of motor and propellers is:

$$
I \omega^{\bullet}=\frac{K_{i}}{R}\left(u-k_{b} \omega\right)-k_{1} \omega^{2}-k_{2} \omega
$$

where $I_{m}$ denotes the moment of inertia around the horizontal axis, $\theta_{1}$ is the elevation angle, $T_{u}$ is the torque of the propulsion force of the main propeller, $T_{f}$ is the friction torque, $T_{m}$ is the torque of the gravitation force of the helicopter body, $\omega$ is the angular velocity of DC motor and I, R, $\alpha, \beta_{11}, \beta_{21}, k_{i}, k_{b}, k_{2}, k_{1}$ are constants. Suppose that $b=\frac{k_{b} k_{i}+R k_{2}}{k_{i}}, a=\frac{k_{1} R}{k_{i}}, I_{r}=\frac{I R}{k_{i}}$. Values of the parameters for the laboratory helicopter are shown in Table1.

TABLE 1

AMOUNT OF PARAMETERS FOR THE HELICOPTER

\begin{tabular}{|c|l||}
\hline \hline Parameter & amount \\
\hline \hline $\mathrm{T}_{\mathrm{g}}$ & 0.0955 \\
\hline$\alpha$ & 0.0687 \\
\hline $\mathrm{a}$ & 0.051 \\
\hline $\mathrm{b}$ & 0.3206 \\
\hline $\mathrm{k}_{1}$ & 0.0297 \\
\hline$\beta_{11}$ & $0.0043 * 10^{\wedge}-4$ \\
\hline$\beta_{21}$ & $1.4178 * 10^{\wedge}-4$ \\
\hline $\mathrm{I}_{\mathrm{m}}$ & 0.0043 \\
\hline & \\
\hline & \\
\hline & \\
\hline & \\
\hline & \\
\hline
\end{tabular}




\begin{tabular}{|l|l|}
\hline $\mathrm{I}_{\mathrm{r} 1}$ & 0.0555 \\
\hline
\end{tabular}

By defining the state variables as $x_{1}=\theta_{1}$, $x_{2}=\dot{x}_{1}=\dot{\theta}_{1}$ and $x_{3}=\omega$, the state space description of elevation direction of the laboratory helicopter is obtained as:

$$
\begin{aligned}
\dot{x}_{1}= & x_{2} \\
\dot{x}_{2}= & \frac{1}{I_{m}}\left(k_{1} x_{3}^{2}-\left(\beta_{11}\left|x_{3}\right|+\beta_{21}\right) x_{2}+T_{C 1} \operatorname{sign}\left(x_{2}\right)-\right. \\
& \left.-T_{g} \sin \left(x_{1}+\alpha\right)\right) \\
& \dot{x}_{3}=\frac{1}{I_{r 1}}\left(u_{1}-a_{1} x_{3}^{2}-b_{1} x_{3}\right)
\end{aligned}
$$

In order to obtain the multiple linear models, the nonlinear state equations are liberalized every 10 degrees. Hence suitable linear models for helicopter have been obtained in full range of the helicopter operating space. The linear models approximate the behavior of the non-linear plant near the operating points. Table 2 shows the set of linear models. Models concern with angles above 90 degree are unstable, which is compatible with the actual performance of the helicopter. In practice, by applying a step signal to the helicopter elevation motor, if the amplitude is low the helicopter settle down in an angle below 90 degree. The helicopter moves upward by increasing the amplitude of the input signal. Above 90 degree, it becomes unstable and jump to 140 degree which is the maximum elevation angle.

\section{B. Evaluation obtained model's performance}

A method for evaluation of an obtained model performance is to design a controller based on that model and to implement the controller to the real plant. If the system's control loop performance is compatible with control criteria, it means that the obtained model is suitable for that plant. Hence for each model a PID controller has been designed. The design criteria are $0 \%$ overshoot and 4 seconds settling time. The result of implementing for 70 and 120 degree is illustrated in figure 3 and 4 . As it is depicted from these figures, the step responses have not overshoot and the settling times are less than 4 seconds. This means that the obtained models have acceptable accuracy.

TABLE2

MULTIPLE MODEL OF HELICOPTER IN DIFFERENT ANGLES

\begin{tabular}{|l|l|}
\hline \hline Angle(Degree) & Transfer Function \\
\hline \hline 60 & $\frac{373.4}{s^{3}+8.741 s^{2}+7.902 s+52.32}$ \\
\hline 70 & $\frac{373.4}{s^{3}+8.741 s^{2}+5.632 s+32.95}$ \\
\hline 80 & $\frac{373.4}{s^{3}+8.741 s^{2}+3.245 s+12.58}$ \\
\hline 90 & $\frac{373.4}{s^{3}+8.741 s^{2}+.8132 s+8.174}$ \\
\hline 100 & $\frac{373.4}{s^{3}+8.741 s^{2}-1.59 s-28.68}$ \\
\hline 110 & $\frac{373.4}{s^{3}+8.741 s^{2}-3.89 s-48.31}$ \\
\hline
\end{tabular}
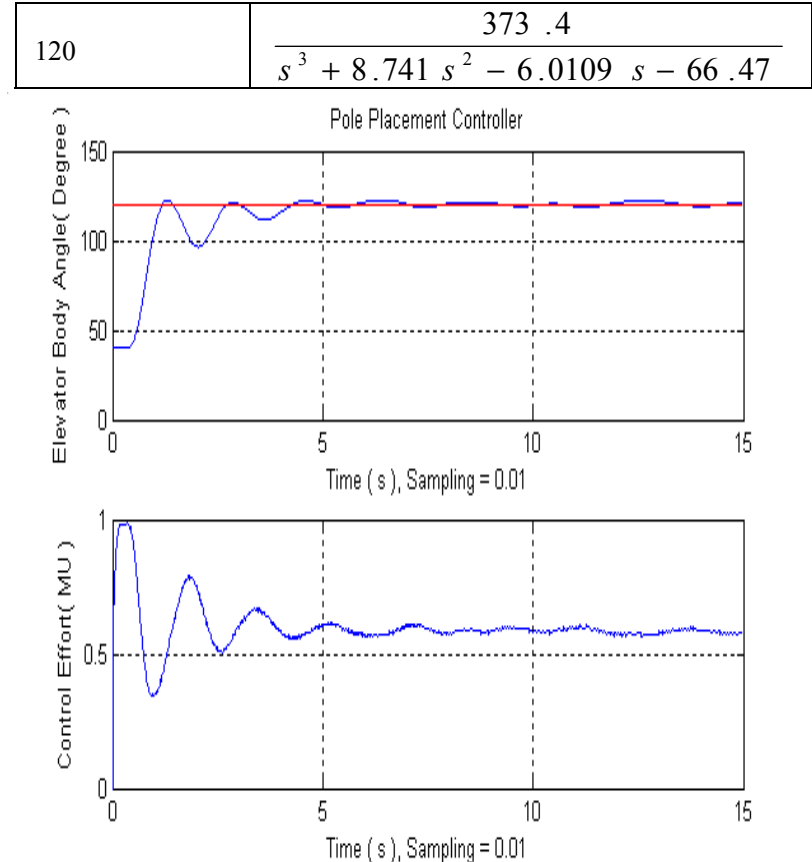

Figure3. Implementation the result of controller designed for 120 degree
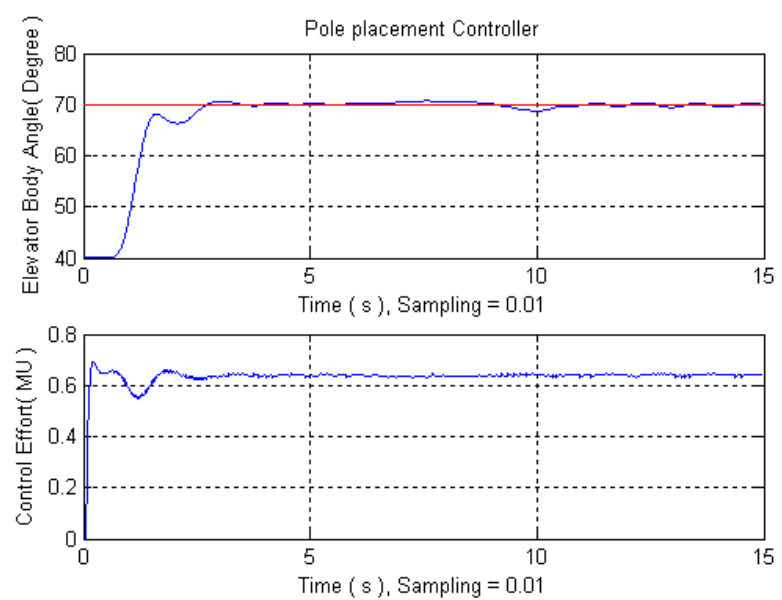

Figure 4. Implementation the result of controller designed for 70 degree

\section{DESIGNINIG MULTIPLE CONTROLLER}

Multiple controllers are designed using pole placement by state feedback, observer for estimation of state variables and integrator to remove steady state error and disturbances. A

Controller is designed for each model. Since one of the three states (process output) is available, thus for simplicity reduced order observer is used [7].

The multiple model controller schemes have been shown in figure 5. This structure has been used because of its suitable control signal, simplicity of its implementation and the possibility of systematically designing controller. Closed loop poles determine the quality of systems output response. Hence, selection of closed loop pole is important to design a controller. To design the controller for each model of the 


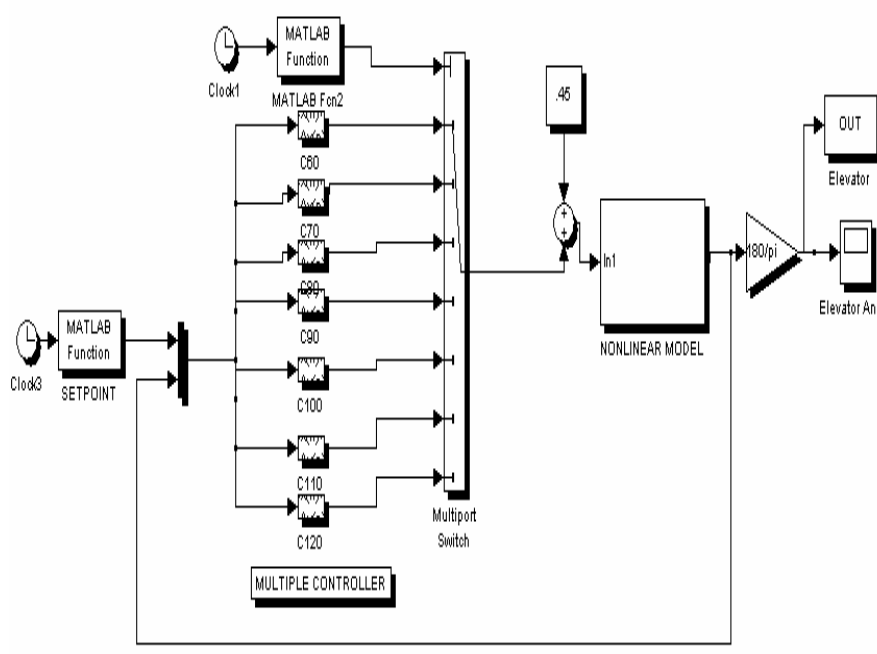

Figure5. The multiple model- controller scheme.

Laboratory helicopter, two design criteria are selected to determine the closed loop poles:

- $\% \mathrm{O} . \mathrm{S}=0$

- $T_{S}<4$ second

For example for 90 degree According to the design

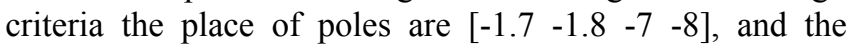
observer poles are selected as $\mathrm{Po}=\left[\begin{array}{lll}-4 & -5 & -6\end{array}\right]$. Pole placement controller structure has been shown in figure 6 .

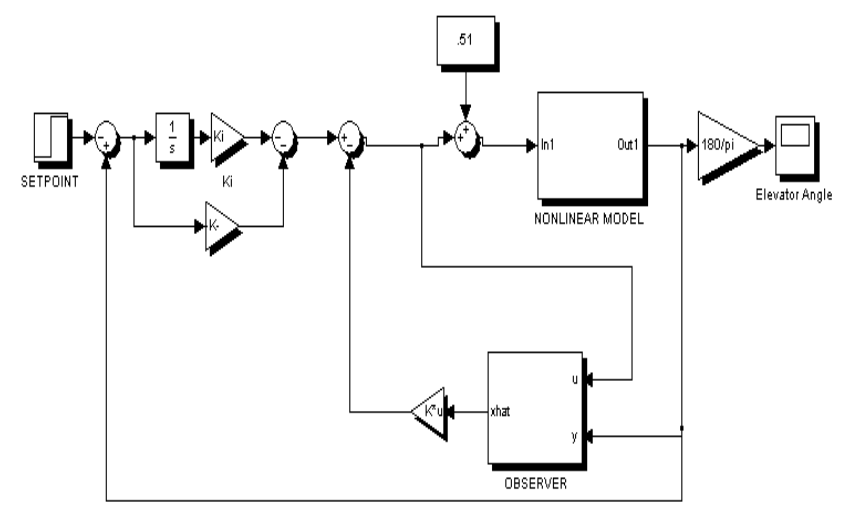

Figure 6: pole placement controller structure

\section{SWITCHING BETWEEN CONTROLLER}

There is a different structure for model estimation and control. In general there are two methods for multiple modeling [8]:

- IMM or interactive multiple modeling: In this method the final control signal is the weighted combination of all or part of the models.

- SMM or switching multiple modeling: In this method the controller relevant to the best model is selected to control the plant in each instant of time.
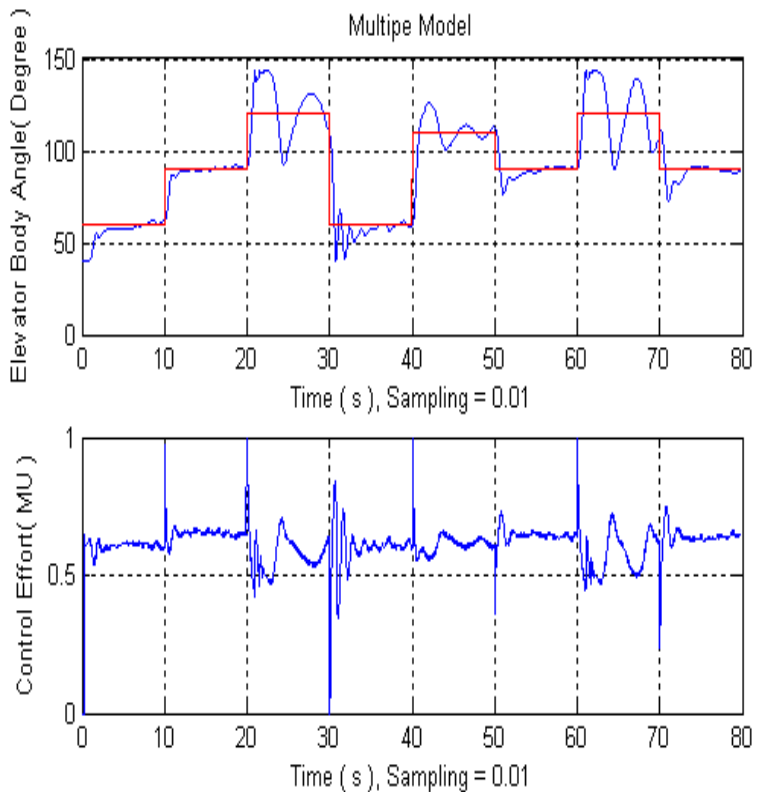

Figure7: The result of applying controller designed for 90 degree to full operating ranges

SMM has been used for multiple modeling of this helicopter. In this method, a new model is selected among the stored multiple models after the plant behavior changes. Then the controller related to the selected model is utilized to control the plant. The multiple modeling method has a better performance than adaptive controller for processes that their dynamic behavior instantaneously changes [6]. Since any changes in laboratory helicopter dynamics can be detected by set point, hence the controller for new situation can be determined by set point and there is no need to compare the process output with the output of all the models in order to pick the best model. This makes the selection faster and more accurate. The pole placement controller is linear hence it has validity in small interval around operating point as the operating point changes its validity decreases. Figure 7 shows a controller designed based on the model of the helicopter in 90 degree but applied in full operating range. As shown in this figure, as the set point changes, this controller has a desired performance for models which are close to 90 degree on the time interval between 10 to 20 seconds and more or less on the time intervals $[50,60]$ and $[70,80]$. However, according to figure 7 , settling time is more than 4 seconds for angles below 90 degree on the time intervals $[0,10]$ and $[30,40]$ seconds and relative stability is degrades for angle more than 90 on the time intervals $[20,30],[40,50]$ and $[60,70]$.

It should be noticed that the laboratory helicopter behavior is different in rising and falling steps. Hence we select the controller based on the following rules. These rules have been obtained based on various tests on the helicopter. Assume that $\theta_{1}$ is initial set point angle and $C_{\theta_{1}}$ is the controller related to that and $\theta_{2}$ is the final set point angle and $C_{\theta_{2}}$ is controller related to this final angle.

If the set point changes from $\theta_{1} \rightarrow \theta_{2}$, then: 


$$
\text { if }\left\{\begin{array}{l}
\theta_{2}>\theta_{1} \Rightarrow C_{\theta_{1}} \rightarrow C_{\theta_{2}} \\
\theta_{2}<\theta_{1} \& \theta_{1}-\theta_{2} \leq 20 \Rightarrow C_{\theta_{1}} \rightarrow C_{\theta_{2}} \\
\theta_{2}<\theta_{1} \& 20<\theta_{1}-\theta_{2} \leq 30 \Rightarrow C_{\theta_{1}} \rightarrow C_{\theta_{2}+10} \\
\theta_{2}<\theta_{1} \& \theta_{1}-\theta_{2}>30 \Rightarrow C_{\theta_{1}} \rightarrow C_{\theta_{2}+20}
\end{array}\right\}
$$

\section{SIMULATION RESULTS}

The performance of multiple model controller methods designed in the previous section is tested on nonlinear dynamic model (1). The result of simulation is presented in figure 8 . This figure shows that design criteria are satisfied using the switching rule (2).

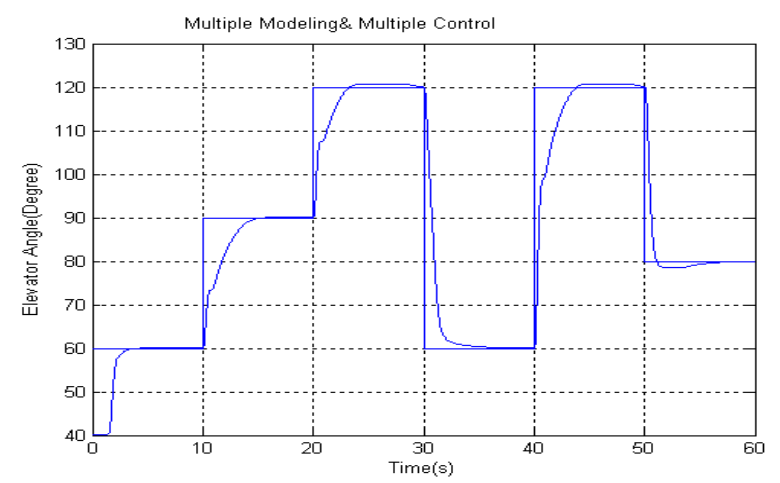

Figure8: Simulation result of multiple model controller

In order to compare the performance of multiple model approach with a single controller, the output of model is presented in figure 9 when the controller designed for 100 degree is applied to the same set point as the one in figure 8 . Also, the output of model is presented in figure 10, when a single PID controller is employed in the closed loop control system. This controller is tuned to have an acceptable performance for all operating points. By comparing figure 8 with figures $9 \& 10$, the superiority of the multiple model controllers is observed. As shown in figure 9, the pole placement controller designed for 100 degree is difficult to prevent the oscillation. On the other hand, as shown in figure 10, in angles more than 100 degree the PID controller has big and variable overshoot in different angles. But the multiple model controllers have a small amount of overshoot only in transient from 120 to 80 degree in the time interval [50, 60]. Moreover, in this case the step response is similar in both stepping up and stepping down variation of set point.

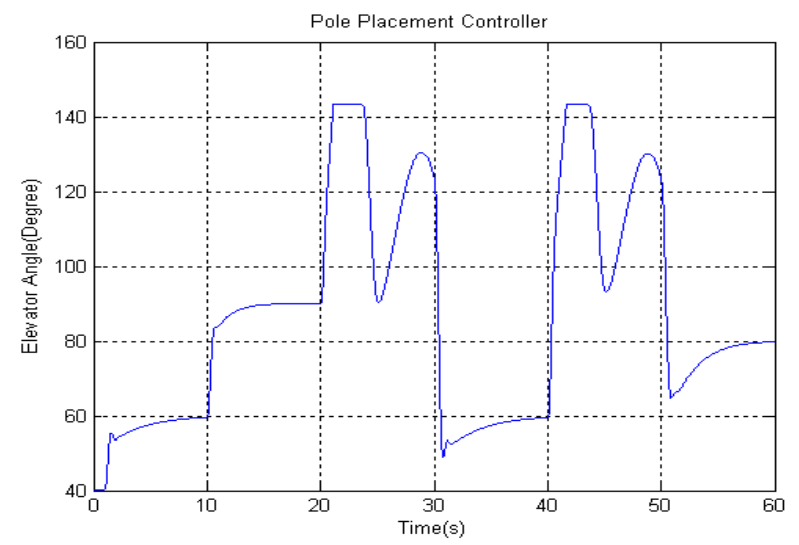

Figure9: simulation result of the controller designed for 100 degree applied to full operating range

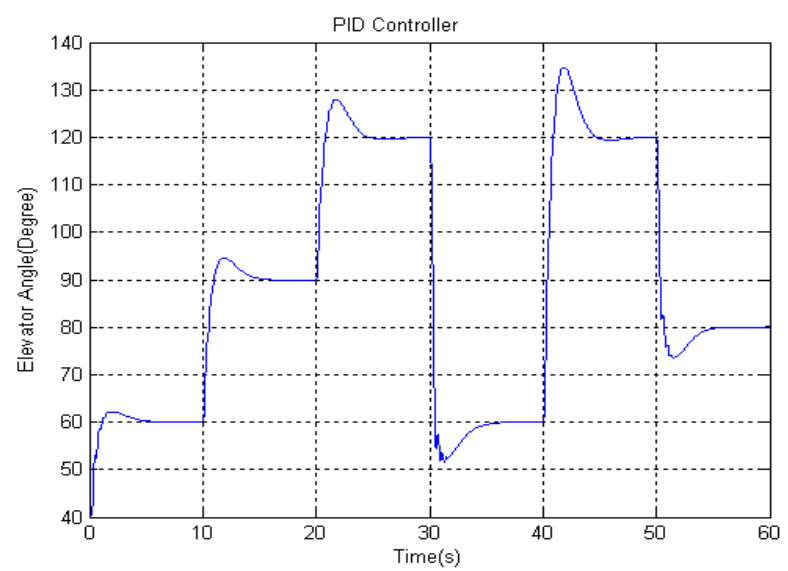

Figure 10: the result of simulation a PID controller to full operating ranges

\section{IMPLEMENTATION OF THE CONTROLLER}

In this section, the designed controller is implemented on the laboratory helicopter. Changing the elevation angle caused to variation the helicopter's dynamical behavior. In order to change dynamic behavior of the helicopter the set point has been changed. The new controller is selected according to rules (2) as the set point changes. One of the main problems in multiple model controllers is to identify the best model for the new situation. Hence, the best model is selected at each instant of time by comparing the behaviors of the models with that of the actual process. The controller of best model, describes the ongoing process, is selected to control the plant. The process of identification of the best model is time consuming specially when there are many models available. It degrades the performance of controller when the plant behavior changes rapidly [9]. In the laboratory helicopter, the new situation can be identified in two ways:

- using the set point

- using the plant output
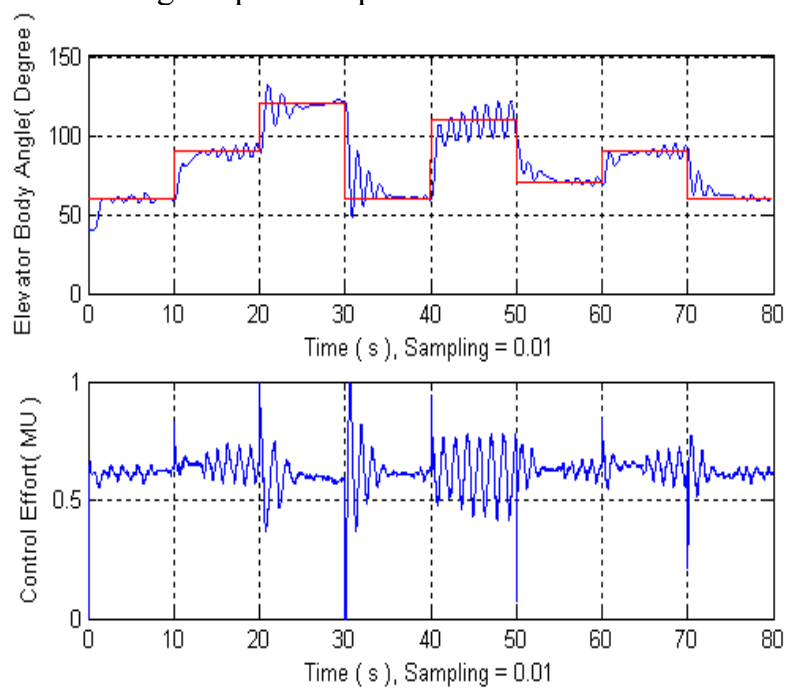

Figure 11: The result of using output for distinguish the new model

Figure 11 shows the helicopter output when its output used to identify the best model. In this case, the output behavior is oscillatory. Since the plant output has noise and disturbance is high in the helicopter, thus the output remarkably changes at each instant of time. Therefore, if it uses to identify the best model, the selected model changes at most of the time and hence the controller switches to new 
controllers rapidly. Variation of controller in short period of time made the control signal to oscillate something that causes the helicopter output to oscillate. On the other hand, using criteria like integral of error to identify
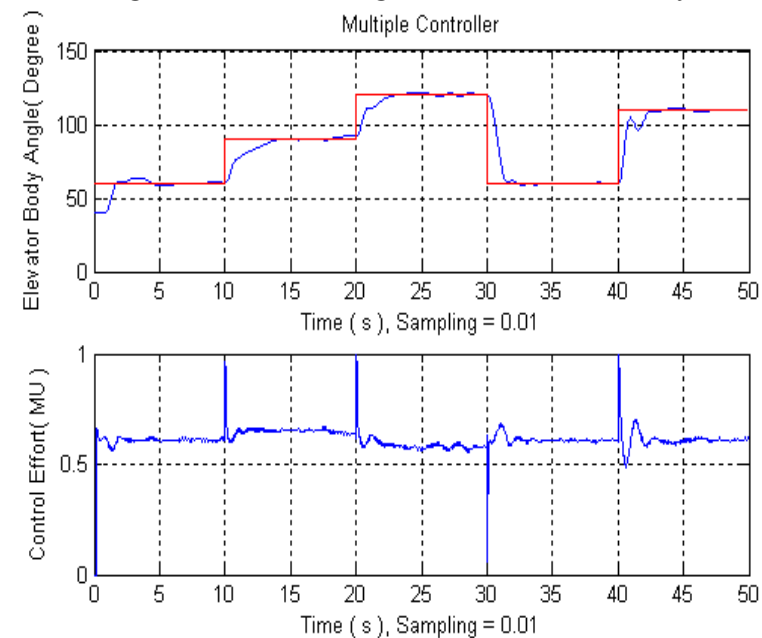

Figure 12: the result of using the set point for distinguish the new model

the best model to limit the number of switches cause some delay that itself ends to oscillation of output. So, we use the set point to recognize the new situation. Figure 12 shows the output behavior when using the set point to select the best controller. By comparing figures 11 and 12 it has been shown that using the set point has a better control performance [10].

In the following, the performance of multiple model controllers is compared to other methods when they are implemented to the helicopter. In figure 13, 14 the result of implementation of the PID controller and a single controller designed by pole placement method has been shown. By comparing these figures to figure 15 , the superiority of the multiple model controller method to satisfying the controller designing criteria is observed.
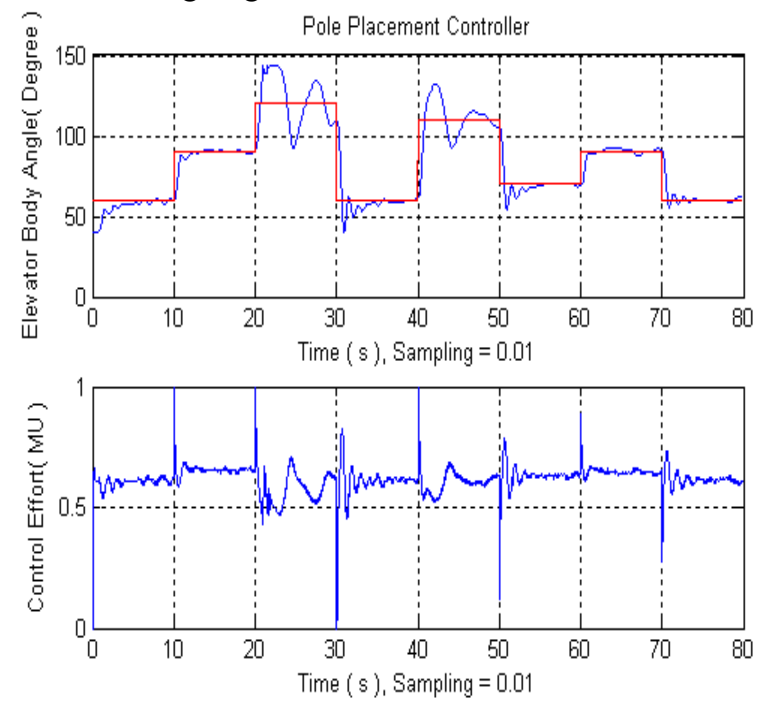

Figure 13: Result of implementation controller design for 90 degree to full operating ranges
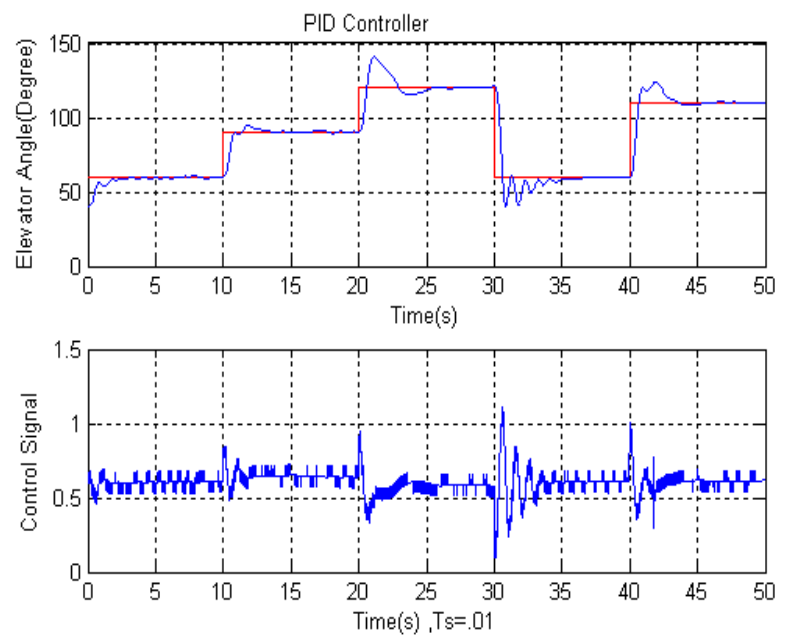

Figure 14: Result of implementation a PID controller to full operating ranges

A nonlinear controller was designed for this helicopter using feedback linearization technique in [11]. Figure 16 illustrates the plant output using this method which outlined as above. By comparing figure 16 and figure 15 improvement of the system output performance with multiple model controllers is showed
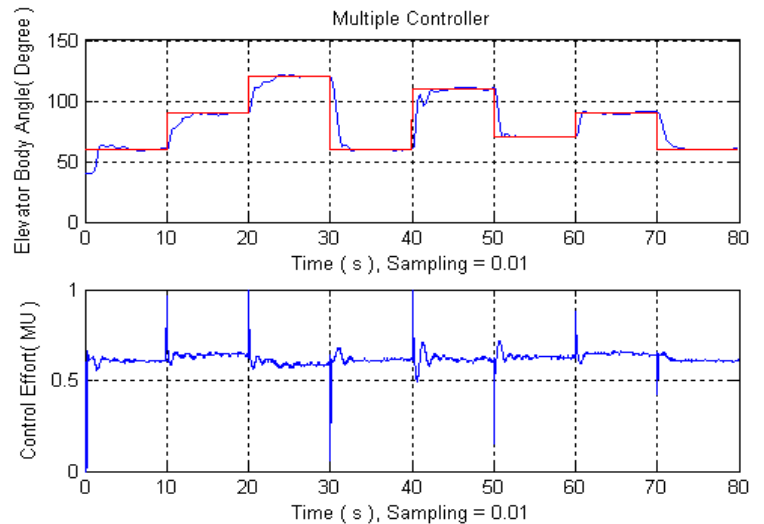

Figure 15: Result of implementation the multiple model-controller to full operating ranges

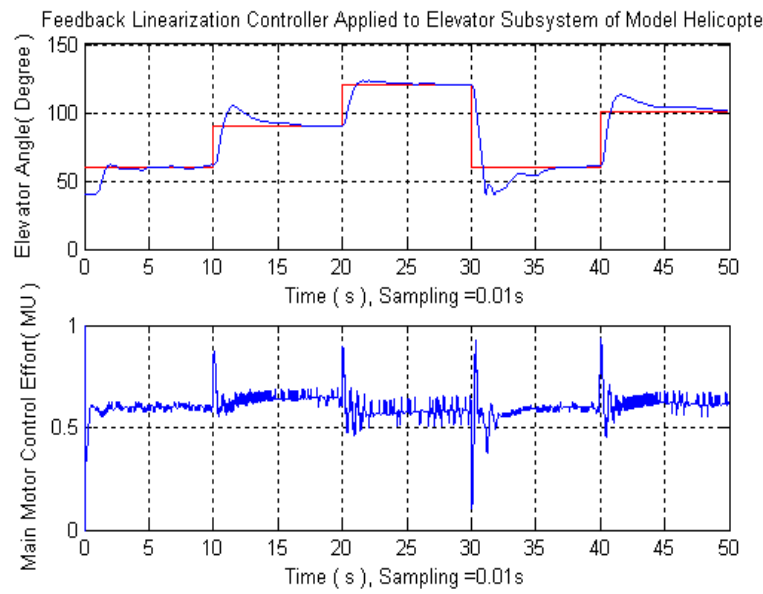

Figure 16: Result of implementation the nonlinear controller to full operating ranges

The output overshoot is about $0 \%$ in figure 15 and the settling time is smaller than that of figure 16 where the settling time is more than 4 seconds and the overshoot is noticeable. 


\section{CONCLUSIONS}

In this paper multiple models are computed by obtaining nonlinear dynamic equations of laboratory helicopter and linearization of the elevation channel equations. Then the multiple controllers are designed for each model by pole placement technique using state feedback controller. The multiple model-controllers are simulated using helicopter nonlinear model then the controller is implemented on the helicopter. The superiority of this technique for rapidly changing systems is investigated by comparison of the multiple model controller approach with other linear or nonlinear methods.

\section{REFERENCES}

[1] Bo" ling, J. M., et al. Multi-model adaptive control of a simulated $\mathrm{pH}$ neutralization process. Control Engineering Practice, 15 (2007) 663-672

[2] Pouya Bashivan , Alireza Fatehi, Ehsan Peymani," Multiple-Model Control of $\mathrm{pH}$ Neutralization Plant Using the SOM Neural Networks",2008 IEEE

[3] Ehsan Peymani, Siavash Fakhimi, Alireza Fatehi \& Ali Khaki Sedigh, "Automatic Learning in Multiple Model Adaptive Control", Proceedings of UKACC Control conference, September 2008.

[4] A.Fatehi," Multiple Modeling of Time-varying Systems by Selforganizing Map Neural Network",PhD Thesis,Tohoku univesity, 2001

[5] Humusoft , "CE150 Helicopter Model User'sGuide"1992.

[6] Michal Hoc,"Helicopter in virtual space", M.Sc Thesis, Lulea University of Technology,Prague ,2008

[7] A. Khaki-Seddigh,"Modern Control Theory",Tehran University Press, 2004

[8] Alireza Fatehi, Kenichi Abe, "Flexible structure multiple modeling using irregular self-organizing maps neural network," International Journal of Neural Systems, Vol. 18, No. 3, 2008, 233-256

[9] Humusoft , "Real Time Tool User'Guide",1992

[10] V. Nazarzehi, "Desigan and implementation of multiple modeling and control approach for Twin Rotor Helicopter", M.Sc Thesis, K.N.T. University,Iran,2003

[11] R.B.Khosroshahi."Desigan and implementation of linear and nonlinear control on Twin Rotor Helicopter", M.Sc Thesis, K.N.T. University,Iran,2001

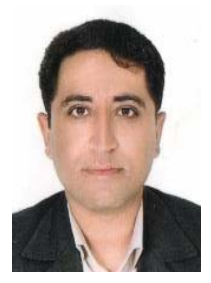

Valimohammad Nazarzehi received his BS degree in Electrical Engineering from Ferdowsi University of Mashhad, Mashhad ,Iran in 2000, the Ms degree in control Engineering from K.N.Toosi University of Technology,Tehran,Iran in $2003 \mathrm{He}$ is currently a lecturer in Chabahar Maritime UniversityHis research interests are multiple modeling and control and system identification.

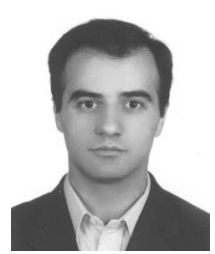

Alireza Fatehi received the BS degree in electronics engineering from Isfahan University of Technology, in 1990, the MS degree in control engineering from Tehran University, Tehran, Iran, in 1995 and Ph.D. degree also in control engineering from Tohoku University, Sendai, Japan, in 2001. Dr. Fatehi is currently an assistant professor of control engineering in control department of K.N. Toosi University of Technology (KNTU), Tehran, Iran and head of Advance Process Automation \& Control (APAC) research group. His research interests include process control systems, intelligent control systems, multiple control systems, nonlinear predictive controllers and fault detection.

Mehdi Zamanian received his BS degree in Electrical Engineering from university of Tehran,Tehran ,Iran ,the Ms degree in control Engineering from K.N.Toosi University of Technology,Tehran,iran, He is currently a lecturer in control department of K.N. Toosi University of Technology (KNTU), Tehran, Iran. His research interests are Linear Control Systems, Multivariate Time Series and and Data Acquisition Circuits. 